\title{
Político desde lo común. Reflexión sobre la posibilidad del posconflicto en Colombia*
}

\author{
The political from the ordinary. \\ Reflection of the possibility of post-conflict in Colombia \\ 0 político a partir do comum. \\ Reflexão sobre a possibilidade do pós-conflito na Colômbia
}

Fecha de entrega: 5 de septiembre de 2015

Fecha de evaluación: 15 de diciembre de 2015

Fecha de aprobación: 15 de enero de 2016

Francisco González Granados**

\section{Resumen}

En el presente escrito plantearé cómo la posibilidad de un escenario de posconflicto en Colombia se puede construir a través de una redefinición de lo político desde un planteamiento de lo común, entendiendo por ello, un modo de política inclusiva que permita construir la relación nosotros/ellos en torno a intereses o valores de los individuos que conforman un grupo con afinidades, intereses

* Este texto pertenece a una serie de escritos que el autor ha venido elaborando en su investigación doctoral sobre el tema la política y la comunidad, de acuerdo con la apuesta por un escenario de inclusión de diferencias conflictivas. DOI: http://dx.doi.org/10.15332/s0120-8462.2016.0114.09

* $\quad$ Estudiante doctorado en Filosofía de la Universidad Nacional, magíster en Filosofía de la misma universidad, licenciado en Filosofía de la Universidad Santo Tomás. Correo electrónico: gragonfra@gmail. com 
disímiles y pasiones antagónicas que pueden ser resueltas en un campo agonístico. Dicho planteamiento lo abordaré en cuatro momentos; el primero, expone el concepto de comunidad de Schmitt; el segundo, deconstruye el concepto schmittiano de lo político; el tercer momento presenta la concepción de lo común como el resultado de las relaciones de multiversos políticos; finalmente, en el cuarto momento, se reflexiona sobre la construcción de un escenario de posconflicto en Colombia, concebido como la relación de multiversos políticos.

Palabras clave: político, conflicto, agonístico, multicultural, común.

\section{Abstract}

With this paper I will raise how the possibility of a post-conflict scenario in Colombia can be built through a redefinition of the political from a perspective of the ordinary, understanding by this a way of inclusive politics that allows to build the relationship us/them around interests or values of individuals within a group with affinities, different interests and conflicting passions that can be resolved in an agonistic field. I will address this approach in four moments; the first one, presents the concept of community of Schmitt; the second, deconstructs the Schmittian concept of the political; the third moment presents the conception of the ordinary as the result of multiverse political relations; finally, the fourth moment, reflects on the construction of a post-conflict scenario in Colombia, regarded as the multiverse political relation.

Keywords: political conflict, agonistic, multicultural, ordinary. 


\section{Resumo}

Neste artigo vou plantear como a possibilidade de um cenário de pósconflito na Colômbia pode ser construído através de uma redefinição do político a partir de um levantamento do comum, entendendo por isto, uma forma de política inclusiva que permita construir a relação nós/ eles em torno de interesses ou valores dos indivíduos que conformam um grupo com afinidades, interesses diferentes e paixões antagónicas que podem ser resolvidas em um campo agonístico. Dito levantamento abordar-se-á em quatro momentos; o primeiro apresenta o conceito de comunidade de Schmitt; o segundo desconstrói o conceito schmittiano do político; o terceiro momento apresenta a concepção do comum como o resultado das relações de multiversos políticos; finalmente, no quarto momento, refletimos sobre a construção de um cenário de pós-conflito na Colômbia, concebido como a relação de multiversos políticos.

Palavras-chave: político, conflito, agonístico, multicultural, comum. 
Schmitt (2009) postula una relación entre la teología y lo político que le permite establecer analogías sistemáticas entre los conceptos teológicos y políticos. A partir de dicha relación Schmitt se da la licencia de transitar de una esfera a otra para analizar la evolución de las ideas filosófico-políticas. Ahora bien, este camino trazado nos proporciona una manera de explorar el concepto de unidad y su función en el pensamiento político.

El autor alemán ilustra la raíz metafísica de la política con la unidad que tiene el cristianismo a partir de Dios, pues, el catolicismo es el mejor ejemplo de dicha unidad, dado que representa la unidad del pueblo de Dios, llamado a salvarse y orientado por el pastor Jesús, quien es representado en la tierra por el Papa. Según el autor alemán, el pensamiento metafísico permite configurar una realidad unificando sus componentes, según un orden -finalidad-, mediante una representación derivada de la autoridad de quien establece el orden -la Idea-. Solo en el pensamiento metafísico es factible in-formar (dar forma) a una serie de factos de acuerdo a una finalidad, es decir, según una relación medios y fines. Igualmente, la política procede acorde a una finalidad concreta, con el presupuesto de la autoridad y la representación, es decir, tanto el pensamiento metafísico como la política pueden dar Forma.

Esta dependencia de la política respecto al pensamiento metafísico es analizada por Schmitt a través de la relación entre la Iglesia católica y el Estado: "la Iglesia (...) presupone junto a sí al Estado político, (...) desea vivir con el Estado en una específica comunidad en la que dos representaciones se contraponen como interlocutoras" (Schmitt, 2000, p. 31). Para Schmitt la Iglesia y el Estado pueden ser interlocutores precisamente porque ambos representan algo en nombre de una "Idea", de donde procede su autoridad. Schmitt señala que ningún sistema político se mantiene solo por la técnica de mantenimiento del poder, sino que se fundamenta en algo más fuerte: "la Idea es parte de lo político, porque no hay política sin autoridad y no hay autoridad sin un ethos de la convicción” (Schmitt, 2000, p. 21).

Schmitt, influenciado por el pensamiento contrarrevolucionario, retoma la tesis de De Maistre sobre la necesidad de una Idea - característica propia de lo religioso- como fundamento del poder político: "todas las instituciones imaginables, si no reposan sobre una idea religiosa, son efímeras. Son fuertes y duraderas en la medida en que están divinizadas" (De Maistre, 1955, p. 124). El autor advierte que la Idea solo opera 
en un plano metafísico ya que esta se presenta como lo trascendente desde lo que se sustenta un orden y es representable en la realidad concreta; mediante la Idea tanto "en el mundo político y moral, como en el mundo físico, existe un orden normal" (De Maistre, 1955, p. 64).

Así, el interés de Schmitt por la forma política del catolicismo, es decir por el ethos de donde deriva su autoridad, lo lleva a afirmar que la Iglesia posee en toda su pureza el pathos de la autoridad en el que se fundamenta su capacidad representativa. La relación entre ethos y pathos es el punto neurálgico al que llega Schmitt, la Idea se presenta con el talante de aquello que está allende, imponiéndose con majestuosidad y dignidad (autoridad), logrando afectar el ánimo (del representante y los destinatarios), $y$, consecuentemente moviéndolos a ciertos tipos de acciones y costumbres. A partir de la Idea se forman valores, surge una moral, cuando esta es representada. Ahora bien, Schmitt especifica cómo se da tal representación en la Iglesia, a saber: "una representación personal y concreta de una personalidad concreta" (Schmitt, 2000, p. 23). De tal modo, para nuestro autor, la Iglesia tiene la capacidad de crear figuras representativas tales como el papa, el emperador, el caballero, el monje, entre otros, dado que la idea de representación está sujeta a la de una autoridad personal que la autorice, de manera que lo representado goza de la autoridad.

Al enfatizar la relación entre la representación y la Idea que la fundamenta, Schmitt, a través del análisis de la idea política del catolicismo, le atribuye a esta la capacidad y la energía de generar una "triple gran forma" que abarca una "forma estética de lo artístico, una forma jurídica del derecho y una forma de poder histórico-universal” (Schmitt, 2000, p. 27), es decir, configura una realidad según un orden. Este orden implica una racionalidad, así, Schmitt señala que la Iglesia católica, configura la realidad acorde a una relación medios-fines, según la Idea de la que deriva su autoridad, y, por lo tanto da una "dirección normativa de la vida social humana" (Schmitt, 2000, p. 15).

Una vez expuesta la capacidad de la Idea del catolicismo de generar un orden, Schmitt centra su atención en cómo esa Idea se mantiene incólume históricamente a pesar de los cambios políticos y bifurcaciones internas. Tal característica lleva al autor a preguntarse cómo definir la Idea política del catolicismo que le ha permitido mantenerse a lo largo de los siglos como institución. La respuesta que plantea se centra en el carácter universalista del catolicismo, derivado de la cosmovisión de una realización práctica de la Idea que informa la realidad fungiendo como epicentro que irradia sus 
características. Para nuestro autor, el carácter del universalismo del catolicismo radica en que la Iglesia católica es una complexio oppositorum que se caracteriza porque:

No parece que haya contraposición alguna que ella no abarque. Desde hace mucho tiempo se gloría de unificar en su seno todas las formas de Estado y de gobierno, de ser una monarquía autocrática cuya cabeza es elegida por la aristocracia de los cardenales, en la que, sin embargo, hay la suficiente democracia para que, sin consideración de clase u origen, como lo formuló Dupanloup, el último pastor de los Abruzos tenga la posibilidad de convertirse en soberano autocrático. Su historia conoce ejemplos de asombrosa adaptación, pero también de rígida intransigencia; de capacidad de resistencia móvil varonil y de flexibilidad femenina; de orgullo y humildad extrañamente mezclados (Schmitt, 2000, p. 8).

La descripción sobre la Iglesia católica como complexio oppositorum se centra en mostrar su capacidad de integración y subsunción de diferentes formas de organización, podría decirse que la esencia de la Iglesia católica es ser la forma de la formas. En los ejemplos utilizados por Schmitt llama la atención la alusión a la dicotomía varonilfemenino, ya que esta expresa la concepción de la Iglesia como una institución con pleno poder de decisión y acción frente a una realidad por in-formar y como una madre que vela y acoge a sus hijos. Tal dicotomía permite la interpretación de la complexio oppositorum como la capacidad de unificar diferencias a través de la creación de ideas que logran la representación de diferentes realidades. A juicio de Schmitt, la esencia de la complexio oppositorum "reside en una específica superioridad formal sobre la materia de la vida humana. (...) Esta particularidad formal del catolicismo consiste en la estricta aplicación del principio de la representación" (Schmitt, 2000, p. 10). El principio de la representación es la expresión de la capacidad de crear formas para organizar y estructurar los diferentes aspectos de la vida humana. A través de la representación se crea una unidad articulada desde una Idea. Schmitt considera que la capacidad de la Iglesia católica de crear representación es el factor del pathos del que procede su autoridad porque la representación permite crear convicción respecto a algo. Dicha capacidad de representación de la Iglesia se ilustra de la siguiente manera: "la Iglesia es hoy el último y solitario ejemplo de la capacidad medieval para formar figuras representativas (el papa, el emperador, el monje, el caballero, el mercader. (...) El vaticano es la última)" (Schmitt, 2000, p. 23). 
En virtud de lo anterior, la Iglesia católica y su universalismo se muestran como la capacidad de generar formas trascendentales que otorgan una forma a los hechos de la vida, tal característica es inherente a la Iglesia dado su talante transcendental derivado de la Idea central: Dios. El universalismo de la Iglesia católica es lo que permite crear una comunidad homogénea, puesto que está cohesionada en torno a la Idea que se hace concreta a través de diferentes figuras representativas. Estas cuando alcanzan un grado de cohesión fuerte se erigen en representaciones con poder de decisión, tal y como es la figura del papa. Una vez erigida una figura representativa con poder de decisión la unión que permite la comunidad - koinonía - en torno a la Idea se torna como La Comunidad frente a otros tipos de agrupaciones o asociaciones, estableciendo rasgos distintivos, tal es el caso de la complexio oppositorum.

Cabe afirmar que el interés de Schmitt por la relación analógica entre la teología y la política se centra en indagar sobre la esencia de la unión que crea una comunidad, alcanzada a través de una Idea que articula la realidad de la vida en una visión trascendental y teleológica, en la que incluso las diferencias hacen parte de un plan que se presenta como el verdadero y único -carácter excluyente y homogeneizante-. El catolicismo romano ofrece un ejemplo valioso, a los ojos de Schmitt, de cómo conformar y mantener una comunidad política en torno a una Idea y figuras representativas que generan un pathos de autoridad y convicción.

\section{II}

En El concepto de lo político, Schmitt señala que es difícil dar una respuesta a la pregunta, ¿qué es lo político? dado que "el campo de relaciones de lo político se modifica incesantemente” (Schmitt, 1991, p. 39). La manera en la que Schmitt delimitó el problema fue estableciendo un criterio que le permitiera distinguir categorías políticas fundamentales que, a su vez, facilitarán una definición conceptual de lo político:

La distinción política específica, aquella a la que pueden reconducirse todas las acciones y motivos políticos, es la distinción amigo y enemigo, (...) es desde luego una distinción autónoma, (...) en el sentido de que ni se funda en una o varias de esas otras distinciones (Schmitt, 1991, p. 56). 
Mediante tal distinción se puede establecer el grado de unión o separación de un grupo humano, su punto máximo de asociación — unidad política- y disociación junto con el grado de identidad que dicho grupo logra frente a otro. Además, "los conceptos de amigo y enemigo deben tomarse (...) en su sentido concreto y existencial. (...) Enemigo es solo un conjunto de hombres que siquiera eventualmente (...) se oponen a otro conjunto análogo" (Schmitt, 1991, p. 58).

El criterio de amigo y enemigo permite identificar las oposiciones y antagonismos más intensos, de modo que si la tensión o conflicto entre dos grupos llegase a ser cercano a la enemistad en grado sumo, entonces, dicho conflicto se podrá considerar como político. Sin embargo, la enemistad no implica la completa desaparición de la otra parte; además, la enemistad puede darse tanto en un plano teórico como en un plano concreto y siempre exige tomar una decisión respecto al otro. El criterio amigo y enemigo permite la exploración de lo político a través de situaciones concretas o agrupaciones que giren en torno a la oposición máxima radical, característica por la que muchas situaciones de otras esferas de la vida humana, en las que surgen oposiciones, son confundidas con lo político, tal es el caso de lo moral (lo bueno y lo malo), lo estético (lo bello y lo feo), entre otras.

Acerca de la consideración schmittiana de lo político, Mouffe precisa que es necesario mantener su carácter polémico, ya que si se niega pueden surgir antagonismos irresolubles que destruyan las políticas democráticas: "[radical democracy] demands that we acknowledge difference the particular, the multiple, the heterogeneous? In effect, everything that has been excluded by the concept of Man in the abstract" (Mouffe, 1993, p. 13). La interpretación de Mouffe sobre lo político permite considerarlo como un espacio antagónico en el que un grupo se define respecto a otro. Según la autora, Schmitt abordó lo político desde lo colectivo ya que el criterio amigo-enemigo identifica una situación como política según el grado de unión y decisión de un grupo frente a otro, inscribiendo lo político en el campo de las identidades relacionales:

In my view one of Schmitt's central insights is his tesis that political indentities consist in a certain type of we/they relation, the relation friend/enemy which can emerge out of very diverse forms of social relations. By bringing to the fore the relational nature of political identities, he anticipates several currents of thought, such as post-structuralism, that will later stress the relational carácter of all identities. Today, thanks to those later theoretical developments, 
we are in a position to elaborate better what Schmitt focefully asserted but left untheorized (Mouffe, 2005, p. 14).

Schmitt abrió el camino para pensar lo político desde las diferentes formas de las relaciones sociales, ya que la finalidad de lo político es: "organizar lo mejor posible las condiciones exteriores y colectivas propias para dar a la unidad política y a los miembros que en ella viven las mejores posibilidades para responder a lo que es, $o$ consideran individualmente, su vocación" (Monereo, 2004, p. 12). Según Mouffe, la circunstancia de pensar lo político a través de diversas formas de las relaciones sociales, invita a encontrar otros modos políticos de construir la relación We/They que permitan mantener a raya o eviten la emergencia del antagonismo, es decir, postular políticas democráticas que permitan la construcción de la relación We/They de una manera diferente a la antagónica:

Once the category of the "enemy" is validated at all, the function of the continuous "challenge" becomes suspect. What does it take for this challenge to be considered "legitimate"? Apparently, for one thing, it must be issued by an adversary who may be on an opposing team but who is definitely playing the same game (Diane, 1999, p. 470).

Lo anterior no implica que se deba eliminar el carácter antagónico de lo político sino que puede ser incorporado en otras formas, como es el caso de concebir lo político como un espacio agonístico. La autora plantea que lo político ha de ser visto como el campo de la confrontación en que las partes enfrentadas tienen algo en común - la disputa por la legitimidad de sus pretensiones y valores- que es la razón de su enfrentamiento. Dicho enfrentamiento no busca la eliminación del otro dado el carácter relacional de lo antagónico. No obstante, el antagonismo tampoco debe ser eliminado a través de la razón deliberativa que busca integrar las partes en conflicto anulando los puntos de diferencias - como lo propone el liberalismo-, ya que lo político sería eliminado al ser lo antagónico su carácter esencial.

La consideración de lo político como un espacio agonístico apunta a reconsiderar la relación We/They menos que una disputa de enemigos y más de adversarios. En esta las partes en conflicto reconocen que no es factible una solución racional a su conflicto; sin embargo sí reconocen la legitimidad de su oponente: "they are adversaries not enemies. This means that, while in conflict, they see themselves as belonging to 
the same political association, as sharing a common symbolic space within which the conflict takes place" (Mouffe, 2005, p. 20). A diferencia de Schmitt, que señaló que lo político radicaba en la tensión entre dos grupos rivales que no tenían nada en común, Mouffe rescata la esencia antagónica de lo político y la ubica como la confrontación acaecida dentro de un grupo o comunidad. Con este desplazamiento, el carácter esencial de lo político es 'domado' o canalizado ya que al situarlo dentro de una comunidad o grupo se hace necesario la creación de diferentes instituciones y prácticas que faciliten el desarrollo del antagonismo de una manera agonística; en palabras de Mouffe (2000): "the potential antagonism can be played out in an agonistic way" (p. 21).

Cabe aclarar que el concepto de adversario de Mouffe no es el mismo que en el discurso liberal que considera lo político como un terreno neutral y un espacio de deliberación pública y libertad en el que los competidores buscan ocupar las posiciones de poder. En cambio, en el espacio agonístico planteado por la autora, los adversarios tienen la intención de cuestionar la hegemonía dominante de la comunidad o grupo con el fin de lograr una transformación radical de las relaciones de poder o lograr nuevas configuraciones:

Another aspect of Mouffe's agonistic framework that shows a rich Cosmopolitan potential is the transformation of enemy into adversary, (...) the scope of democratic politics and institutional arrangements is to construct a "them" as an adversary. The institutional arrangements are not enough for this transformation and it should be completed by a radical change within political identity, or conversión (Caraus, 2016, p. 101).

Lo agonístico es concebido, entonces, como una lucha entre grupos con proyectos hegemónicos opuestos en un espacio común y bajo unas reglas de aceptación, en caso del triunfo de alguno de los grupos, es decir, se impone la hegemonía de un grupo pero no se elimina la del otro que en cualquier momento puede derrotar al grupo hegemónico de turno. Lo agonístico es la manera de sublimar lo antagónico de lo político dentro del espacio simbólico común de un grupo en el que coexisten varios proyectos hegemónicos y no uno solo como en el caso de lo político planteado por Schmitt. 


\section{III}

Algunas de las reflexiones actuales sobre lo político y la democracia han resaltado el desafío de pensar las comunidades políticas en torno al pluralismo cultural. Al respecto, desde la perspectiva de la confrontación agonística se puede abordar la pregunta por la relación entre el pluralismo cultural y las comunidades políticas. Sin embargo, antes de abordar dicha pregunta cabe precisar que no toda comunidad política es equivalente a un grupo cultural y que dentro de una comunidad política pueden coexistir diferentes grupos culturales. El pluralismo cultural tiene un carácter transversal, es decir, la posibilidad de la existencia de un mismo grupo cultural en diferentes comunidades políticas. Ahora bien, un grupo cultural no se reduce únicamente a rasgos o características étnicas, sino que también puede ser el producto de la convergencia de diversos aspectos que giran en torno a un sentido o visión del mundo tales como género, orientación sexual, discapacidades, entre otros.

El pluralismo cultural plantea un reto para cualquier comunidad política, puesto que se presenta como un conjunto de diversas visiones del mundo: "there are a number of incommensurable values and corresponding ways of life which are all legitimate objects of human aspiration, and that different citizens can make different, but equally legitimate choices within that set" (Weinstock, 2002, p. 242). El desafío que representa el pluralismo cultural a cualquier comunidad política es la capacidad institucional para articular los diferentes intereses y aspiraciones de los grupos culturales que la conforman, con el fin de garantizar los derechos de cada uno de los ciudadanos pertenecientes a diferentes grupos culturales.

A través de la propuesta de Mouffe sobre lo político como un espacio agonístico, se puede concebir la comunidad política como el resultado de las relaciones de convergencia, divergencia y pugna de los intereses de los grupos culturales. Estas relaciones se dan en un espacio común formalizado mediante prácticas institucionales. El objetivo de cada grupo es alcanzar el poder de cambiar dichas prácticas institucionales acorde a sus intereses y valores como grupo:

Important to the constitution of political community and to the formation of a radical democratic hegemony is the creation of what Mouffe calls a "chain of equivalence" among different struggles (for example, feminism, gay rights, and anti-racism). A chain of equiva- lence does not form in terms of a common 
essence shared by different groups but through determining the adversary, the "them" to which "we" are opposed (Worsham \& Olson, 1999, p. 165).

Es claro que el concepto de comunidad política adquiere un carácter heterogéneo que conlleva un grado de cohesión frágil e incierta. Sin embargo, el elemento que dinamiza las relaciones entre los diversos grupos heterogéneos es la necesidad del contrincante dado el carácter relacional de las identidades. El espacio agonístico adquiere sentido, precisamente, cuando la actividad de diversos grupos es efectiva. Por otra parte, la esencia y posibilidad de las actividades políticas radican en la esencia antagónica de lo político:

Lo político puede adquirir su fuerza de los más diversos ámbitos de la vida humana; de contraposiciones religiosas, económicas, morales y otras. No indica a una esfera de acción en particular sino tan solo al grado de intensidad de una asociación o disociación de personas cuyas motivaciones pueden ser de índole religiosa, nacional (tanto en sentido étnico como cultural), económica, etc. pudiendo estas motivaciones producir diferentes uniones y divisiones en distintas épocas (Schmitt, 1991, p. 42).

Acorde a la cita, la comunidad política que deriva Schmitt es de carácter homogéneo, una comunidad cerrada que sublima sus disensiones internas mediante la identificación de un enemigo. No obstante, según Mouffe, estas disensiones internas se pueden sublimar, sin la necesidad de acudir a un enemigo externo, mediante la concepción de lo político como un espacio agonístico, dando así un carácter heterogéneo a la comunidad política que pasaría de ser un universo homogéneo que suprime las voces divergentes internas a un conjunto de multiversos que dinamizan la esfera política en virtud de sus intereses contrapuestos, pero, sin caer en la ilusión de una anulación o solución final del antagonismo a través de una discusión racional, como lo propone el liberalismo. La imposibilidad de lograr una solución final al antagonismo que permita la consolidación de una comunidad política radica en el carácter pasional del ser humano que irreductible a cualquier forma racional de organización política.

Precisamente, la concepción agonística de lo político mantiene el carácter pasional propio del antagonismo. El valor de la propuesta de Schmitt sobre lo político como un espacio de conflicto radicó en vincular lo político como una dimensión constitutiva del ser humano ligada a las pasiones. Dicha dimensión no puede ser anulada 
en ningún momento, tal y como lo expresa Mouffe con la necesidad de mantener el conflicto pero de una manera sublimada. El error de Schmitt fue considerar que una vez articulada la unidad política mediante la identificación de un enemigo común se centraba todas las disensiones internas hacia el exterior, dado que las pasiones tienen un carácter impredecible y voluble dando posibilidad a la emergencia y expresión de múltiples diferencias. En consecuencia, la posibilidad de una comunidad política universal y homogénea adquiere un carácter utópico. Desde la propuesta agonística, la comunidad política adquiere un carácter heterogéneo que se sustenta en el desafío de crear mecanismos racionales -ciencia política- que más que reprimir, logren sublimar las pasiones facilitando espacios en que se manifiesten y choquen con otras, es decir, la característica común de los diferentes grupos culturales en una comunidad política es su lucha por la configuración de las relaciones de poder, a través de esta lucha la comunidad política puede ser vista como el espacio vital del desarrollo de los intereses y valores de los grupos políticos.

\section{IV}

La lucha de los grupos culturales por el poder, dentro de una comunidad política, se centra en alcanzar el poder para transformar las instituciones de la comunidad política según sus intereses. Esta lucha se establece como el vínculo en común entre los diferentes grupos culturales contenidos dentro de una comunidad política. Lo común aparece desde dos perspectivas. La primera alude al espacio de posibilidad de confrontación: la comunidad política que será considerada como el espacio agonístico en el que los intereses de diversos grupos se desarrollan. La unidad lograda por la comunidad política se daría gracias a la necesidad del otro o de los otros como posibilitadores de la construcción de la identidad de un grupo dado: "we must institutionalize a true pluralism, a pluralism of subjects. (...) We transcend a certain individualistic notion of rights and (...) elaborate a notion of solidarity" (Mouffe, 2001, p. 101).

Así, la relación We/They no solo es una dicotomía sino que expresa también un conjunto: el conjunto de los intereses de We frente al conjunto de los intereses de They que articulan un espacio de posibilidad y realización. La comunidad política vista desde la perspectiva agonística no sería homogénea sino heterogénea, en donde hay lugar incluso para las disidencias y los disensos. Precisamente, la segunda perspectiva para considerar lo común se relaciona con la lucha y confrontación de 
intereses dentro de una comunidad política como el factor dinamizador y creador de lo político. Lo común en la confrontación de intereses radicaría en el carácter pasional que impulsa la defensa y consecución de estos. La dinámica de lo político radica en el carácter conflictivo y antagónico del ser humano, derivado del carácter incierto e impredecible de las pasiones. Si se anularan las pasiones, se erradicaría la esencia de lo político y la comunidad política sería inconcebible porque no habría necesidad de establecer canales, métodos e instrumentos formales - ciencia política- para procurar la convivencia y seguridad humana.

La imposibilidad de erradicar las pasiones, en cierta medida, garantiza la existencia de la comunidad política y su vínculo común radicado en la lucha por configurar las relaciones de poder. Sobre estas, cabe precisar, que deben tener un carácter horizontal en el espacio agonístico para procurar conservar el carácter heterogéneo de la comunidad política porque en el caso contrario - relaciones verticales - se pasaría a la imposición de un grupo y la anulación de los demás generando una situación de autoritarismo o totalitarismo en el que se crea la ficción de una comunidad homogénea.

Siguiendo con esta línea de interpretación la posibilidad de una comunidad homogénea adquiere un carácter utópico, ya que en términos de la vida política de una comunidad y de las dinámicas de los grupos culturales, contenidos en esta, la construcción y fijación de identidades es contingente dado el carácter pasional del ser humano: "that identities are in fact the result of processes of indentifications and that they can never be completely fixed. We are never confronted with we/they oppositions expressing essentialist identities pre-existing the process of identification" (Mouffe, 2005, p. 18). El carácter pasional del ser humano le imprime a lo político un elemento de irreductibilidad que constituye el combustible de la vida política de una comunidad, de modo que la hegemonía impuesta por un grupo en una comunidad política, concebida como un espacio agonístico, no es perenne sino está sujeta a ser derrotada en cualquier momento ya que lo político consiste en mantener 'el juego' por reconfigurar las relaciones de poder según los intereses de los diferentes grupos. 


\section{Referencias}

Caraus, T. (2016). Towards an agonistic cosmopolitanism: Exploring the cosmopolitan potential of Chantal Mouffe's agonism. Critical Horizons, 17(1), 94-109. Recuperado de http://eds.a.ebscohost.com.ezproxy.unal.edu.co/

De Maistre, J. (1955). Consideraciones sobre Francia. Madrid: Ediciones Rialp.

Diane, D. (1999). Agonizing [with] Chantal Mouffe. JStor, 19(3), 465-476. Recuperado de http://www.jstor.org/stable/20866256

Monereo, P. J. (2004). El espacio de lo 'político' en Carl Schmitt. En El Leviathan en la teoría del Estado de Tomas Hobbes. Granada: Comares.

Mouffe, C. (1993). The return of the Political. London: Verso.

Mouffe, C. (2001). Hegemony and socialist strategy: Towards a radical democratic politics. London: Verso.

Mouffe, C. (2005). On the political. New York: Routledge.

Schmitt, C. (1991). El concepto de lo político. Madrid: Alianza.

Schmitt, C. (2000). Catolicismo y forma política. Madrid: Tecnos.

Schmitt, C. (2009). Teología política. Madrid: Trotta.

Weinstock, D. (2002). Citizenship and pluralism. En Simon, R. The Blackwell Guide to Social and Political Philosophy. USA: Blackwell.

Worsham, L. \& Olson, G. (1999). Rethinking political community: Chantal Mouffe's liberal socialism. JStor, 19(2), 163-199. Recuperado de http://www.jstor.org/ stable/20866234 